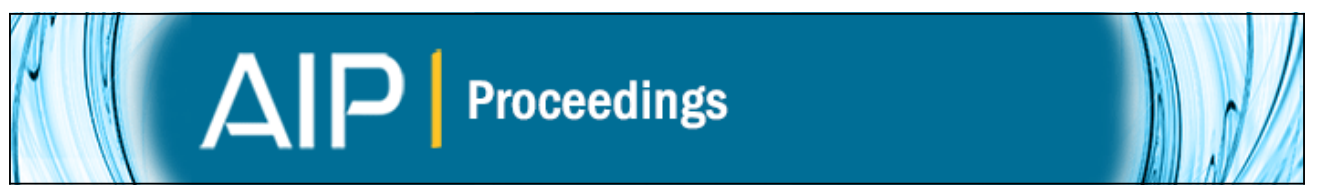

\title{
Surface wave accelerator based on silicon carbide: theoretical study
}

S. Kalmykov, D. Korobkin, B. Neuner, and G. Shvets

Citation: AIP Conference Proceedings 1086, 538 (2009); doi: 10.1063/1.3080964

View online: http://dx.doi.org/10.1063/1.3080964

View Table of Contents:

http://scitation.aip.org/content/aip/proceeding/aipcp/1086?ver=pdfcov

Published by the AIP Publishing

\section{Articles you may be interested in}

Laser-ion acceleration through controlled surface contamination

Phys. Plasmas 18, 040702 (2011); 10.1063/1.3574532

Direct plasma injection scheme in accelerators (invited)a)

Rev. Sci. Instrum. 79, 02B314 (2008); 10.1063/1.2821590

Feasibility of Using Laser lon Accelerators in Proton Therapy

AIP Conf. Proc. 740, 414 (2004); 10.1063/1.1843524

Intense ion beams accelerated by ultra-intense laser pulses

AIP Conf. Proc. 611, 199 (2002); 10.1063/1.1470305

Development of a high-current laser ion source for induction accelerators

Rev. Sci. Instrum. 71, 1216 (2000); 10.1063/1.1150437 


\title{
Surface wave accelerator based on silicon carbide: theoretical study
}

\author{
S. Kalmykov, D. Korobkin, B. Neuner III, and G. Shvets \\ The Department of Physics, The University of Texas at Austin, One University Station C1500, \\ Austin, Texas 78712
}

\begin{abstract}
Compact near-field solid-state accelerating structure powered by a carbon dioxide $\left(\mathrm{CO}_{2}\right)$ laser is considered. The accelerating luminous transverse magnetic mode is excited in a few-micron wide evacuated planar channel between two silicon carbide ( $\mathrm{SiC}$ ) films grown on silicon ( $\mathrm{Si}$ ) wafers. Laser coupling to this mode is accomplished through the properly designed Si gratings. Operating wavelength is dictated by the frequency-dependent dielectric permittivity of $\mathrm{SiC}$ and the channel width. The geometric loss factor $\kappa$ of the accelerating mode is computed. It is found that the unwanted excitation of the guided modes in $\mathrm{Si}$ wafers reduces the laser coupling efficiency and increases the fields inside the Si wafer.
\end{abstract}

Keywords: silicon carbide, planar accelerating structure

PACS: 41.75.Lx, 41.20.Jb, 42.81.Qb, 42.82.Et

\section{INTRODUCTION}

Modern lasers [1] can effectively power ultrahigh-gradient ( $>100 \mathrm{MV} / \mathrm{m}$ ) solid-state accelerator structures [2]. To reduce the risk of breakdown [3], which is the highest for the structures containing metallic parts $[4,5]$, we have proposed earlier $[6,7]$ the design which is based entirely on semiconductors and exploits the unique optical properties of $\mathrm{SiC}$. The sketch of this near-field closed type structure - a sub-wavelength vacuum gap between thin $\mathrm{SiC}$ films epitaxially grown on $\mathrm{Si}$ wafers [8] - is shown in Fig. 1. The structure can be inexpensively manufactured using standard micro-fabrication techniques $[6,7]$.

High tolerance of $\mathrm{SiC}$ to heat loads and electric breakdown (DC breakdown threshold $\sim 300 \mathrm{MV} / \mathrm{m}$ ) makes it the material of choice for radio-engineering devices working in hostile conditions [9]. SiC has a negative dielectric permittivity in the operating range of commercial $\mathrm{CO}_{2}$ lasers $(\lambda \sim 10.6 \mu \mathrm{m})$. The accelerating cavity surrounded by flat $\mathrm{SiC}$ surfaces can thus support a near-luminous transverse magnetic (TM) mode with a fewhundred $\mathrm{MV} / \mathrm{m}$ accelerating gradient. This mode can be interpreted as a double-interface surface wave quite similar to that occurring at the plasma/vacuum interface in the hollow channel plasma wakefield accelerator [10]. It can be excited from the side as shown in Fig. 1 using gratings etched on the top of each wafer [6, 7]. For a sufficiently wide vacuum gap, we find that the Ohmic loss of the mode is so low (and the propagation distance so long) that it can be end-coupled. We refer to this structure as the Surface Wave Accelerator Based on SiC (SWABSiC). 


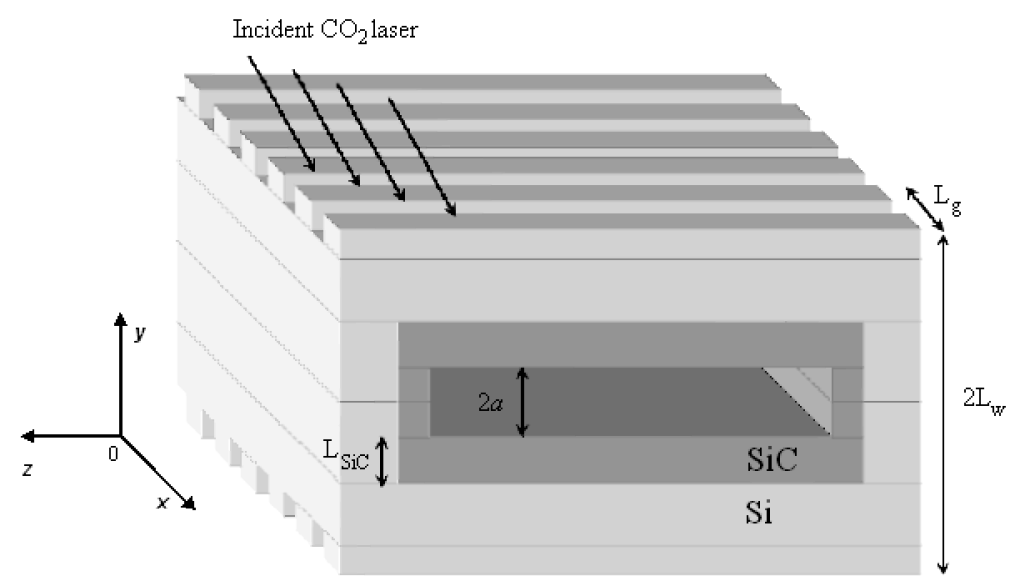

FIGURE 1. Schematic of the SiC-based accelerator. A thin SiC film is grown in the trench at the bottom of a Si wafer. Grating is etched on top of the wafer. The grating couples the incoming mid-IR radiation to the structure. The two wafers are bonded to each other making a symmetric structure with a sub-wavelength opening between the $\mathrm{SiC}$ films. Electrons are accelerated in the $x$-direction. Sample dimensions: $L_{\mathrm{g}}=6 \mu \mathrm{m}$, grooves are $5 \mu \mathrm{m}$ tall, $L_{\mathrm{SiC}}=2 \mu \mathrm{m}$, and wafer thickness is from 20 to $400 \mu \mathrm{m}$.

\section{ANALYTIC PROPERTIES OF THE ACCELERATING MODES}

First, we analytically investigate the dispersion properties of an electromagnetic mode of an idealized SWABSiC: the structure consisting of a flat evacuated channel between two infinitely thick walls of the cubic 3C-SiC polytype [8] with the complex permittivity

$$
\varepsilon_{\mathrm{SiC}}(\lambda)=\varepsilon_{\mathrm{SiC}}^{\prime}+i \varepsilon_{\mathrm{SiC}}^{\prime \prime}=6.5\left[1-\left(\lambda / \lambda_{L}\right)^{2}+i \gamma \lambda\right]\left[1-\left(\lambda / \lambda_{T}\right)^{2}+i \gamma \lambda\right]^{-1},
$$

where $\lambda_{L}=10.29 \mu \mathrm{m}, \lambda_{T}=12.56 \mu \mathrm{m}$, and $\gamma=510^{-4} \mu \mathrm{m}^{-1} . \varepsilon_{\mathrm{SiC}}^{\prime}(\lambda)<0$ for $\lambda_{L}<\lambda<$ $\lambda_{T}$, where $\lambda=2 \pi / k_{0}$ and $k_{0}=\omega / c$. Figure 2(a) shows $\varepsilon_{\mathrm{SiC}}^{\prime}$ and $\varepsilon_{\mathrm{SiC}}^{\prime \prime}$ in the operation range of $\mathrm{CO}_{2}$ laser with ${ }^{13} \mathrm{C}$ isotope fill [7].

A planar waveguide with a symmetry plane $y=0$ supports an accelerating transverse magnetic (TM) mode with a symmetric electric field component $E_{x}$ (accelerating gradient) and anti-symmetric $H_{z}$ and $E_{y}$. Inside the channel, $H_{z}(|y|<a)=$ $[\sin (u y) / \sin (u a)] e^{i \omega t-i k_{x} x}$, while in the wall $H_{z}(|y|>a)= \pm e^{-q(|y|-a)+i \omega t-i k_{x} x}$ (the upper sign stands for $y>a)$. The electric field components are $E_{x}=(c / i \omega \varepsilon) \partial H_{z} / \partial y$, and $E_{y}=-\left(c k_{x} / \omega \varepsilon\right) H_{z}$. Dispersion equation relates the complex propagation constant $k_{x}=k_{x}^{\prime}+i k_{x}^{\prime \prime}$ (longitudinal wave number and the longitudinal attenuation rate) to the mode frequency $\omega$. Using the continuity of $H_{z}$ and $E_{x}$ at the interfaces $y= \pm a$, writing out the wave equation, and recognizing that $k_{x}$ is the same in each medium (Snell's law), we obtain a transcendental equation that can be solved numerically to find $u(\omega)$, and, hence, $k_{x}^{2}=k_{0}^{2}-u^{2}$. The wave is super-luminous $\left(v_{\mathrm{ph}_{x}}=\omega /\left|k_{x}^{\prime}\right|>c\right)$ when $u \approx u^{\prime}$. The mode becomes sub-luminous $\left(v_{\mathrm{ph}_{x}}<c\right)$ when $u \approx i u^{\prime \prime}$. This is only possible if $\varepsilon_{\mathrm{SiC}}^{\prime}<-1$, and

$$
k_{0} a>k_{0} a_{\mathrm{lum}}=-\varepsilon_{\mathrm{SiC}}^{\prime}\left(1-\varepsilon_{\mathrm{SiC}}^{\prime}\right)^{-1 / 2} \text {. }
$$



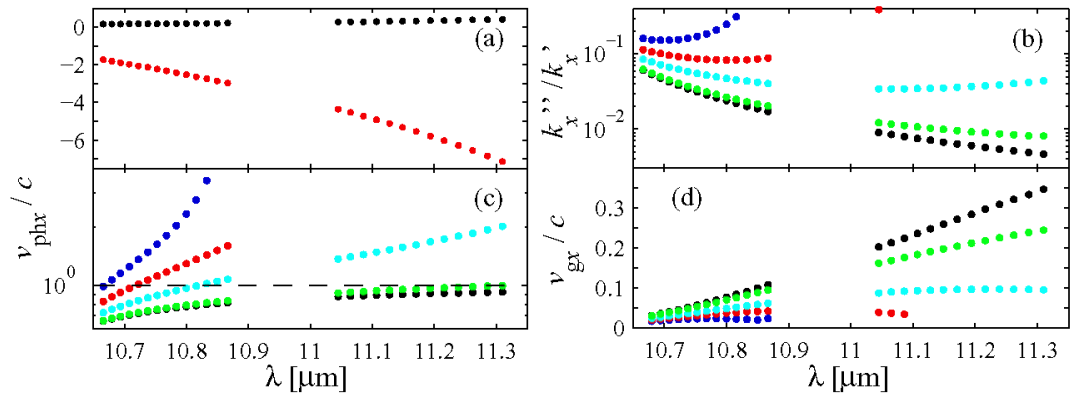

FIGURE 2. (Color online) (a) $\varepsilon_{\mathrm{SiC}}^{\prime}$ (red) and $\varepsilon_{\mathrm{SiC}}^{\prime \prime}$ (black) for the operation wavelengths of the ${ }^{13} \mathrm{CO}_{2}$ laser. (b) Ratio of the spatial decrement to the propagation constant, (c) normalized longitudinal phase and (d) group velocity, $v_{\mathrm{g}_{x}}=\partial \omega / \partial k_{x}^{\prime}$, of the accelerating TM mode. The gap size is $2 a=3.6 \mu \mathrm{m}$ (blue), $4 \mu \mathrm{m}$ (red), $4.8 \mu \mathrm{m}$ (cyan), $9 \mu \mathrm{m}$ (green). Black dots: $a=\infty$.

The equality corresponds to $u=0$ and $v_{\mathrm{ph}_{x}}=c$. This guided TM mode can be interpreted as a superposition of two surface waves ( $\mathrm{SW}$ ) supported by the individual $\mathrm{SiC}$-vacuum interfaces. It is desirable for the energy of the accelerating electric field to be a significant fraction of the total energy. Hence, the ratio $\Delta W=\int_{-a}^{a}\left|E_{y}\right|^{2} d y / \int_{-a}^{a}\left|E_{x}\right|^{2} d y \approx\left(k_{0} a\right)^{2} / 3$ should not be much larger than unity, which implies the sub-wavelength channel width. Numerical examples of the next section correspond to $\lambda=11.31 \mu \mathrm{m}\left(\varepsilon_{\mathrm{SiC}}^{\prime}=-7.14\right)$, which corresponds to the channel width $a_{\text {lum }}=4.5 \mu \mathrm{m}$ and $\Delta W \approx 2.1$.

The dispersion equation is numerically solved for the operating wavelengths $\lambda$ [and the corresponding $\left.\varepsilon_{\mathrm{SiC}}(\lambda)\right]$ of the ${ }^{13} \mathrm{CO}_{2}$ laser. The results are shown Figs. 2(b-d). Both phase and group velocities are almost unaffected by the low resistive losses. It is found that the modes with $\lambda>10.85 \mu \mathrm{m}$ cannot propagate in the channels narrower than $a=3 \mu \mathrm{m}$ because for the long wavelengths $\mathrm{SiC}$ behaves, essentially, as a conductor, and the cutoff condition of $a>\lambda / 4$ applies. Reducing the channel width also increases the losses: $k_{x}^{\prime \prime} / k_{x}^{\prime}>0.1$ for $a=1.8 \mu \mathrm{m}$. Wider channels and longer wavelengths correspond to longer propagation distances $L_{\mathrm{prop}}=1 / k_{x}^{\prime}$. For example, for $\lambda=11.31 \mu \mathrm{m}$ and $a=4.5 \mu \mathrm{m}$ it is found that $L_{\text {prop }} \approx 200 \mu \mathrm{m}$. Therefore, radiation can be end-coupled into the SWABSiC. The advantage of end-coupling is that for thick SiC films the amount of radiation leaking into the $\mathrm{Si}$ wafer can be very small. Unfortunately, for shorter wavelengths and narrower channels resistive losses limit $L_{\text {prop }}$ to tens of microns. Therefore, maintaining a larger accelerating distance requires side-coupling which is the focus of this paper.

\section{LEAKY EIGENMODES OF THE GRATING-COUPLED SWABSIC}

In the side-coupling scenario, a grating etched on the $\mathrm{Si}$ wafer side of the SWABSiC excites leaky accelerating modes inside the vacuum channel of the SWABSiC as shown in Fig. 1. The grating with a pitch $L_{\mathrm{g}}$ splits an incident planar EM wave with a longitudinal wave number $k_{0 x}=k_{0} \sin \alpha$ into a set of diffractive orders (quasi-planar waves) with the wave numbers $k_{m x}=k_{0 x}+2 \pi m / L_{\mathrm{g}}, m=0, \pm 1, \ldots$. Inside the wafer, the number of 

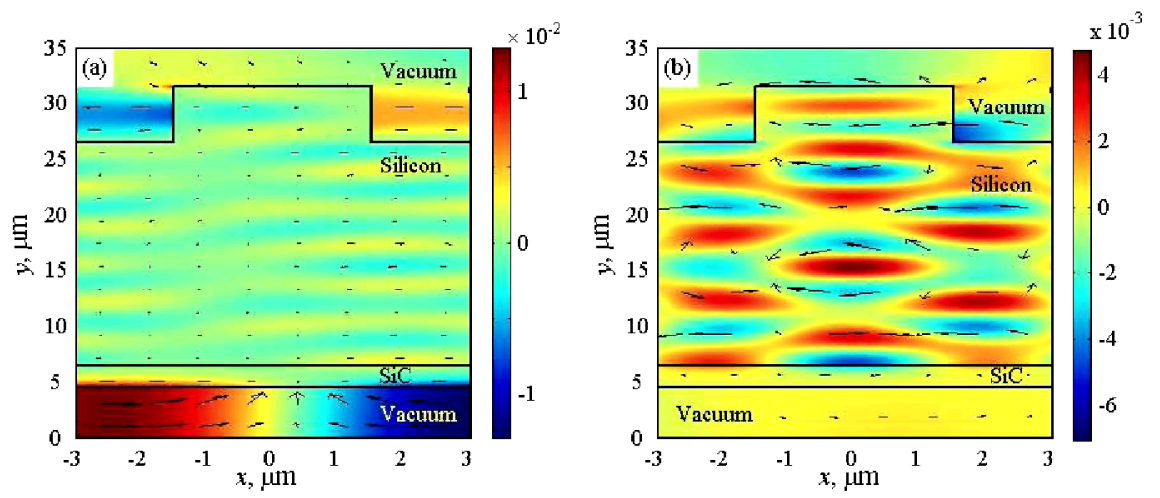

FIGURE 3. (Color online) Electric field (in arbitrary units) corresponding to the anti-symmetric TM resonances in the SWABSiC (one grating period shown). Color: $E_{x}$, arrows: electric field vectors. Plot (a) corresponds to the core mode $\left(\alpha_{1 u m}=-62.25^{\circ}\right)$, plot (b): to the cladding mode at $\alpha=-71.5^{\circ}$.

propagating orders is limited due to a requirement $\left|k_{m_{x}}\right| \leq \sqrt{\varepsilon_{\mathrm{Si}}} k_{0}$. Radiation of various diffractive orders can penetrate from wafer ("cladding") into the vacuum gap ("core") through the thin $\mathrm{SiC}$ films. When the incidence angle satisfies the relation

$$
\sin \alpha_{\mathrm{lum}}=1-m \lambda / L_{\mathrm{g}}, \text { or } k_{m_{x}}=k_{0},
$$

the $m$ th diffractive order can power a luminous accelerating mode. For $\lambda=11.31 \mu \mathrm{m}$, and $L_{\mathrm{g}}=6 \mu \mathrm{m}$, the luminous angle $\alpha_{\mathrm{lum}} \approx-62.25^{\circ}$ corresponds to $m=1\left(\alpha_{\mathrm{lum}}<0\right.$ means the reversal of the longitudinal radiation flux inside the structure). It is expected that for thick SiC coupling between the core and the cladding is weak and that the channel width $a=a_{\text {lum }}$ from Eq. (2) can still be used.

Efficient excitation of the accelerating modes of the structure is achieved when the frequency of the laser is equal to that of the "leaky" eigenmode of the SWABSiC. Those are calculated using the finite element electromagnetic code FEMLAB [11]. We seek the modes with a real part of frequency equal to the frequency of incident radiation. The following geometric and physical parameters are assumed: $\varepsilon_{\mathrm{Si}}=12, \lambda=11.31 \mu \mathrm{m}$ (hence, $\left.\varepsilon_{\mathrm{SiC}}=-7.14+0.4 i\right), a=a_{\mathrm{lum}}=4.5 \mu \mathrm{m}, L_{\mathrm{g}}=6 \mu \mathrm{m}$. To facilitate the interpretation of the simulations results, a thin wafer $(20 \mu \mathrm{m})$ is assumed. In full agreement with the analytic theory, the luminous quasi-longitudinal core eigenmode is found exactly at $\alpha_{\mathrm{lum}}=-62.25^{\circ}$. Electric field distribution corresponding to this resonance is plotted in Fig. 3(a). The complex eigenvalue $\omega \equiv \omega^{\prime}+i \omega^{\prime \prime}$ contains information about both resistive (dominant) and radiative (secondary) losses of the mode. Purely radiative losses can be found by setting $\varepsilon_{\mathrm{SiC}}^{\prime \prime}=0$, resulting in the radiative $Q_{\mathrm{rad}}=2000$. The total $Q$ calculated with the Ohmic losses taken into account is $Q_{\text {tot }} \equiv \omega^{\prime} / \omega^{\prime \prime} \approx 143$ indicating that $Q_{\text {Ohm }} \ll Q_{\text {rad }}$. Purely radiative losses of this mode are almost negligible, while the attenuation rate appears to be nearly the same as for the closed waveguide [see Fig. 2(b), data point for $\lambda=11.31 \mu \mathrm{m}, a=4.5 \mu \mathrm{m}]$.

Coupling of the external radiation to the accelerating mode can be quantified by a loss factor $\kappa$ which relates the peak accelerating gradient $E_{x \max }$ to the energy stored 

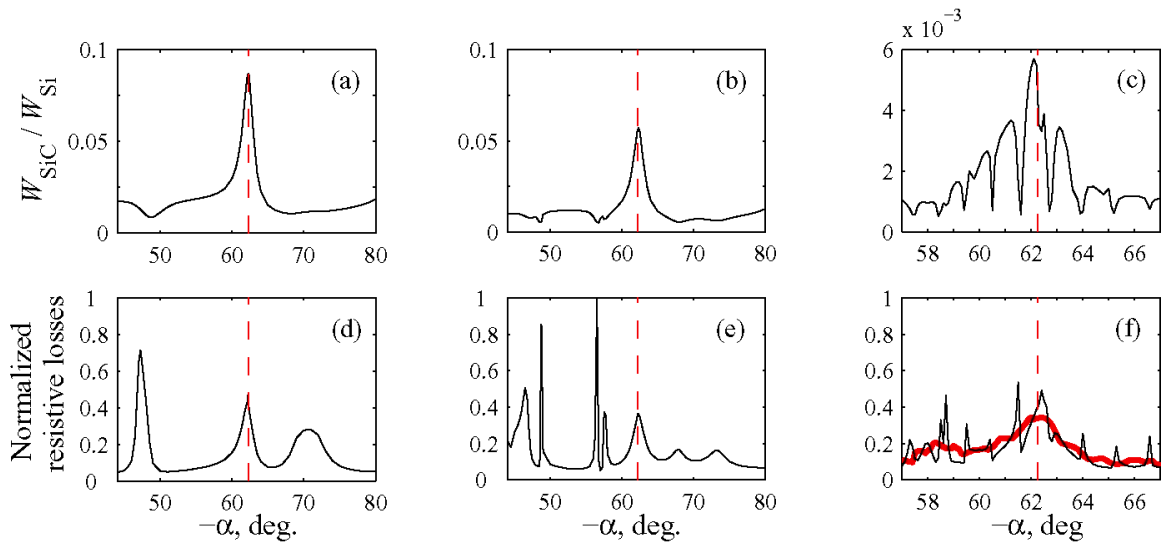

FIGURE 4. (Color online) Top: relative energy content in $\mathrm{SiC}$ film and $\mathrm{Si}$ wafer under laser illumination. Bottom: normalized energy losses. The luminous angle $\alpha_{1 u m}=-62.25^{\circ}$ is indicated by the red dashed line. Wafer thickness is $20 \mu \mathrm{m}(\mathrm{a}, \mathrm{d}), 40 \mu \mathrm{m}(\mathrm{b}, \mathrm{e})$, and $400 \mu \mathrm{m}(\mathrm{c}, \mathrm{f})$. The peak around $\alpha_{\mathrm{lum}}$ corresponds to the excitation of the luminous accelerating mode in the vacuum gap. Other spikes are produced by the excitation of the leaky modes of the Si wafers. Averaging over $\pm 1^{\circ}$ in the vicinity of each data point filters out the spikes produced by the wafer modes [thick red curve in plot (f)].

per unit length in the structure $U$ by $\kappa=E_{x \max }^{2} /(4 U)$ [10]. This is a purely geometric factor independent on the mechanism of the mode excitation. The field structure shown in Fig. $3(\mathrm{a})$ is characterized by $\kappa_{\mathrm{lum}} \approx 0.36 \mathrm{MV} /(\mathrm{pC} \mathrm{m})$, which is almost independent on the wafer thickness. If the accelerating gradient $E_{x \max } \approx 300 \mathrm{MeV} / \mathrm{m}$ is required, $U_{\text {lum }}=E_{x_{\max }}^{2} /\left(4 \kappa_{\text {lum }}\right) \approx 0.0625 \mathrm{~J} / \mathrm{m}$. If the laser illuminates just a few $\mathrm{mm}$ area, the deposited energy must be of order $0.610^{-4}$ Joules.

\section{SIMULATIONS OF THE LASER-DRIVEN SWABSIC}

In this Section we present the results of simulating a laser-driven SWABSiC structure and relate these results to the leaky eigenmode simulations described above. The incident radiation beam is modelled as a planar TM wave. The normalized energy loss is shown in Figs. 4(d-f) for 20, 40, and $400 \mu \mathrm{m}$ thick wafers. We also display in Figs. 4(a-c) the quantity $W_{\mathrm{SiC}} / W_{\mathrm{Si}}=\int_{\mathrm{SiC}}|\mathbf{E}|^{2} d x d y / \int_{\mathrm{Si}}|\mathbf{E}|^{2} d x d y$, which is proportional to the ratio of electric energy density in SiC film and Si wafer in one grating period. Peak in $W_{\mathrm{SiC}} / W_{\mathrm{Si}}$ is associated with the desirable excitation of the surface mode confined to the vacuum channel. The dips correspond to the undesirable excitation of the cladding modes that produce strong fields inside the $\mathrm{Si}$ wafer. Correlation between the peak of absorption ( $\sim 40 \%$ ) and high energy density in SiC occurs only for $\alpha=\alpha_{\text {lum. }}$. This peak of absorption is few-degrees wide because of the relatively high Ohmic losses. The angular width agrees with the simple theoretical estimate $\Delta \alpha \approx\left(\omega^{\prime \prime} / \omega^{\prime}\right)\left(c / v_{\mathrm{g}}\right)\left(1 / \cos \alpha_{\text {lum }}\right) \approx 3.5^{\circ}$. By comparing the simulated electric field profile with that obtained in the eigenmode simulations [Fig. 3(a)], we find a close agreement between the two. The peak accelerating gradient is found to be three times higher than $\left|E_{x}\right|$ in free space. 
Multiple narrow peaks in Figs. 4(d-e) are associated with the eigenmodes of the Si wafers. The number of these modes per given $\alpha$ interval is proportional to the wafer thickness (one peak per $\Delta \alpha \approx 1^{\circ}$ for $400 \mu \mathrm{m}$ wafer). According to Figs. $4(\mathrm{a}-\mathrm{c})$, they correspond to high electric energy density in the Si wafers rather than in $\mathrm{SiC}$ films [one example is displayed in Fig. 3(b)]. Laser beam coupling to these parasitic modes reduces the efficiency of launching the accelerating mode [7] and increases the risk of breakdown in $\mathrm{Si}$, where the DC breakdown limit is estimated to be well below that of $\mathrm{SiC}[9,12])$. Thus, if the strong coupling to the wafer modes cannot be avoided, the accelerating gradient is limited to a few hundreds of $\mathrm{MV} / \mathrm{m}$. Earlier experiments did not show any sharp peaks in the transmission-reflection measurements [7]. That could be attributed to the angular divergence of the focused laser $\left(\approx 1^{\circ}\right)$. Figure $4(f)$ shows that the averaging indeed filters narrow peaks out leaving much wider feature associated with the accelerating mode almost unaffected.

\section{CONCLUSIONS}

Electromagnetic properties of the accelerating modes of the SWABSiC were studied analytically and numerically. The accelerating mode in the vacuum gap between two $\mathrm{SiC}$ films grown on the $\mathrm{Si}$ wafers is powered by the $\mathrm{CO}_{2}$ laser beam. The accelerating cavity is a high quality resonator with a relatively low geometrical loss factor. Hence, moderate laser powers are needed to produce an accelerating gradient of a few hundreds $\mathrm{MV} / \mathrm{m}$. One type of mode excitation (side-coupling through the grating etched in the wafer) was considered. Side-coupling is the only approach for very narrow $(<\lambda / 2)$ gaps. Excitation of the guided modes in the Si wafers increase both launching losses and the risk of the breakdown inside the Si wafer. Future work will focus on several alternative approaches to the mode excitation: end-coupling into a wider channel and excitation through the prism. This work is supported by the U.S. Department of Energy contracts DE-FG02-04ER54763 and DE-FG02-04ER41321.

\section{REFERENCES}

1. I. V. Pogorelsky et al., IEEE J. Quantum Electron. 31, 556 (1995).

2. R. B. Palmer, N. Baggett, J. Claus et al., AIP Conf. Proc. 130, 234 (1985).

3. D. H. Whittum, AIP Conf. Proc. 472, 72 (1999).

4. J. Rosenzweig, A. Murokh, and C. Pellegrini, Phys. Rev. Lett. 74, 2467 (1995).

5. L. C. Steinhauer and W. D. Kimura, Phys. Rev. ST Accel. Beams 6, 061302 (2003).

6. G. Shvets and S. Kalmykov, AIP Conf. Proc. 737, 983 (2004).

7. S. Kalmykov, O. Polomarov, D. Korobkin et al., Phil. Trans. R. Soc. A 364, 725 (2006).

8. C. A. Zorman, A. J. Fleischman, A. S. Dewa et al., J. Appl. Phys. 78, 5136 (1995).

9. Ch. Weitzel, J. W. Palmour, C. H. Carter, Jr. et al., IEEE Trans. Electron Devices 43, 1732 (1996).

10. C. B. Schroeder, D. H. Whittum, and J. S. Wurtele, Phys. Rev. Lett. 82, 1177 (1999).

11. The code is developed and supported by COMSOL, Inc. (1 New England Executive Park, Ste. 350, Burlington, MA 01803, USA).

12. P. P. Pronko, P. A. VanRompay, C. Horvath et al., Phys. Rev. B 58, 2387 (1998). 